\title{
PROJECTIVITY OF THE WHITEHEAD SQUARE
}

\author{
DUANE RANDALL
}

Abstract. We show that the Whitehead square of the generator of $\pi_{n}\left(S^{n}\right)$ is not a projective homotopy class for any integer $n$ with neither $n$ or $n+1$ being a power of 2 .

1. Introduction. Let $\omega_{n} \in \pi_{2 n-1}\left(S^{n}\right)$ denote the Whitehead square of the generator of $\pi_{n}\left(S^{n}\right)$. To determine the integers $n$ for which $\omega_{n}$ can be halved is an important problem in homotopy theory. A homotopy class $[f] \in \pi_{m}(X)$ is called projective in [7] iff $f$ is homotopic to $g \circ \pi$ for some map $g: P^{m} \rightarrow X$ where $\pi: S^{m} \rightarrow P^{m}$ denotes the double covering. We remark that $\omega_{n}$ can be halved iff there exists $g: P^{2 n-1} \rightarrow S^{n}$ with $[g \circ \pi]=\omega_{n}$ and $[g \circ i]=0$ where $i: P^{2 n-2} \subset P^{2 n-1}$. In this note we shall prove the following.

THEOREM. $\omega_{n}$ is not projective for any positive integer $n$ with neither $n$ nor $n+1$ a power of 2 .

The proof consists of constructing a complex with a nontrivial cup product mod 2 if $\omega_{n}$ is projective and then applying decomposability of Steenrod squares to obtain a contradiction.

Note that $\omega_{n}=0$ for $n=1,3,7$ and so is trivially projective. By [5] $\omega_{4}$ is projective, but $\omega_{4}$ can not be halved. Since $2 \pi_{m}(X)$ consists of projective classes for odd $m$ and any space $X$ by [7], $\omega_{15}$ and $\omega_{31}$ can be halved (see [3]) and so are projective.

2. Notation. The coefficient group for cohomology is $Z_{2}$ whenever omitted. We write $H^{*}\left(P^{\infty}\right)=Z_{2}[\alpha]$ and recall $\mathrm{Sq}^{i} \alpha^{j}=\left(\begin{array}{c}j \\ i\end{array}\right) \alpha^{i+j}$. The following result on binomial coefficients is needed.

Lemma. Let $n=2 s+2^{t}$ with $0<s<2^{t-1}$. If $\left(\begin{array}{c}2^{t}-1-i \\ 2 s-2 i\end{array}\right)$ is odd with $0<i \leqq s$, then $\left(\begin{array}{c}n+i-1 \\ n-i\end{array}\right)$ is even.

Proof. Let $\alpha(m)$ denote the number of l's in the dyadic expansion of $m$. Now $\alpha(2 s-i)=\alpha(2 s-2 i)+\alpha(i)$ since the dyadic expansions of $i$ and $2 s-2 i$ are disjoint by hypothesis. If $\left(\begin{array}{c}n+i-1 \\ n-i\end{array}\right)$ is odd, $\alpha(2 s+i-1)=\alpha(2 s-i)+$ $\alpha(2 i-1)$. Thus $\alpha(2 s+i-1)=\alpha(2 s-2 i)+\alpha(i)+\alpha(2 i-1)$, a contradiction.

Received by the editors January 17, 1973 and, in revised form, February 12, 1973.

AMS (MOS) subject classifications (1970). Primary 55E15, 55E40; Secondary 55G10.

Key words and phrases. Whitehead product, projective homotopy class, Steenrod square, Hopf construction. 
3. Proof. Assume $\omega_{n}$ is projective. We obtain the following diagram of horizontal and vertical Puppe sequences with homotopy commutative squares.

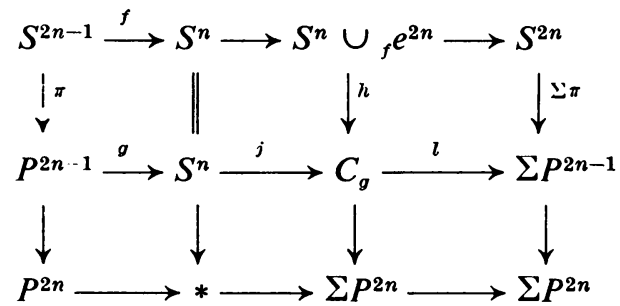

Here $[f]=\omega_{n} . C_{g}$ denotes the mapping cone of $g$ which exists by hypothesis.

THEOREM A. $\omega_{n}$ is not projective for $n=2 s+2^{t}$ with $0<s<2^{t-1}$.

Proof. Assume $\omega_{n}$ projective and let $u$ generate $H^{n}\left(C_{g} ; Z\right)=Z$ in (3.1). Note $j^{*} u$ generates $H^{n}\left(S^{n} ; Z\right)$ and $h^{*} u$ generates $H^{n}\left(S^{n} \cup_{f} e^{2 n} ; Z\right)$. Now $\omega_{n}$ has Hopf invariant \pm 2 and $\Sigma \pi$ induces multiplication by 2 on integral cohomology in dimension $2 n$. Thus $u \cup u$ generates $H^{2 n}\left(C_{g} ; Z\right)$ so $\mathrm{Sq}^{n} u \neq 0$. By [1]

$$
\mathrm{Sq}^{n}=\mathrm{Sq}^{2 s} \mathrm{Sq}^{2^{t}}+\sum_{i=1}^{s}\left(\begin{array}{c}
2^{t}-1-i \\
2 s-2 i
\end{array}\right) \mathrm{Sq}^{n-i} \mathrm{Sq}^{i} .
$$

If $\left(\begin{array}{c}2 t-1-i \\ 2 s-2 i\end{array}\right)$ is odd, $\mathrm{Sq}^{n-i}\left(\sum \alpha^{n+i-1}\right)=\left(\begin{array}{c}n+i-1 \\ n-i\end{array}\right) \sum \alpha^{2 n-1}=0$ by the Lemma so $\mathrm{Sq}^{n-i} \mathrm{Sq}^{i} u=0$. Also $\mathrm{Sq}^{2 s}\left(\sum \alpha^{n+2^{t}-1}\right)=0$ so $\mathrm{Sq}^{n} u=0$, contradiction.

THEOREM B. $\omega_{n}$ is not projective for any odd integer $n$ with $n+1$ not a power of 2.

Proof. Assume $\omega_{n}$ projective so (3.1) exists and write $m=n+1=2 s+2^{t}$ with $0<s<2^{t-1}$. Note that $H^{n}\left(C_{g} ; Z\right)=Z \oplus Z_{2}$. Let $\psi: S^{n} \times S^{n} \rightarrow S^{n} \cup_{f} e^{2 n}$ be the natural map identifying axes. Set $F=h \circ \psi$ '. The morphism $F^{*}$ : $H^{2 n}\left(C_{g} ; Z\right) \rightarrow H^{2 n}\left(S^{n} \times S^{n} ; Z\right)$ is multiplication by 2 and so $F^{*}$ is trivial with $Z_{2}$ coefficients in dimension $2 n$. The Hopf construction applied to $F$ gives a map $\rho: S^{2 n+1} \rightarrow \Sigma C_{g}$ with mapping cone $X$. Sq ${ }^{m}$ is nontrivial on $H^{m}(X)$ by [4]. (See [6] for a complete proof.) Consider the following diagram of cofibrations and homotopy commutative squares.

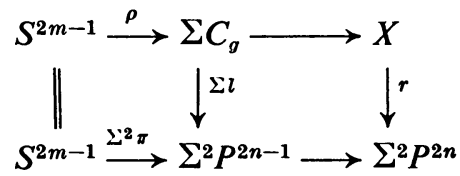


We now apply (3.2). Note that $\mathrm{Sq}^{i}$ is trivial on $H^{m}(X)$ for odd $i$ since $\mathrm{Sq}^{i}=\mathrm{Sq}^{1} \mathrm{Sq}^{i-1}$ and $\mathrm{Sq}^{1}\left(\sum^{2} \alpha^{m+i-3}\right)=0$. Suppose $\left(\begin{array}{c}2^{t}-1-i \\ 2 s-2 i\end{array}\right)$ is odd with $i$ even. We must show $\mathrm{Sq}^{m-i}\left(\sum^{2} \alpha^{m+i-2}\right)=0$ so that $\mathrm{Sq}^{m-i} \mathrm{Sq}^{i}$ is trivial on $H^{m}(X)$ via $r^{*}$ in (3.3). In $H^{*}\left(P^{\infty}\right) \mathrm{Sq}^{m-i}\left(\alpha^{m+i-2}\right) \cup \alpha=\mathrm{Sq}^{m-i}\left(\alpha^{m+i-1}\right)=0$ by the Lemma so $\mathrm{Sq}^{m-i}\left(\alpha^{m+i-2}\right)=0$. Also $\mathrm{Sq}^{2 s}\left(\alpha^{m+2^{t}-2}\right)=0$ so $\mathrm{Sq}^{m}$ is trivial on $H^{m}(X)$, contradiction.

\section{REFERENCES}

1. J. Adem, The iteration of the Steenrod squares in algebraic topology, Proc. Nat. Acad. Sci. U.S.A. 38 (1952), 720-726. MR 14, 306.

2. I. M. James, On the homotopy type of Stiefel manifolds, Proc. Amer. Math. Soc. 29 (1971), 151-158. MR 43 \#1184.

3. —_ Note on Stiefel manifolds. I, Bull. London Math. Soc. 2 (1970), 199-203. MR 41 \#9286.

4. - On the decomposability of fibre spaces, The Steenrod Algebra and its Applications (Proc. Conf. to Celebrate N. E. Steenrod's 60th Birthday, Battelle Memorial Inst., Columbus, Ohio, 1970), Lecture Notes in Math., vol. 168, Springer, Berlin, 1970. MR 43 \#4038.

5. E. Rees, Symmetric maps, J. London Math. Soc. (2) 3 (1971), 267-272. MR 43 \#6923.

6. E. Thomas, On functional cup-products and the transgression operator, Arch. Math. 12 (1961), 435-444. MR 26 \#6972.

7. P. Zvengrowski, Skew linear vector fields on spheres, J. London Math. Soc. 3 (1971), 625-632.

Department of Mathematics, University of Notre Dame, Notre Dame, Indiana 46556

Current address: Pontificia Universidade Católica, Rio de Janeiro, Brazil 